\title{
A qualitative, process-parameter-based model for the release agent transfer during CFRP-part production: an approach to increase the initial bondability
}

\author{
David Blass ${ }^{*} \mathbb{D}$, Sven Hartwig and Klaus Dilger
}

${ }^{*}$ Correspondence:

d.blass@tu-braunschweig.de

Institute of Joining

and Welding, Technische

Universität Braunschweig,

Langer Kamp 8,

38106 Brunswick, Germany

\begin{abstract}
Adhesive bonding shows a high potential to join carbon fiber reinforced plastics (CFRP), if the adhesion between adhesive and part surface is verified. Unfortunately, this adhesion is often reduced in case of the mold-based part production and its unavoidable application of release agents. Due to the resulting presence of release agent residues on the surface of the cured CFRP-parts the adhesion is lowered. To increase the bondability and correlating production efficiency, the amount of release agent transfer should be as small as possible, to reduce the effort in terms of bonding pre-treatment. To achieve this goal, a theoretical, qualitative model about the influence of the relevant processing parameters for a thermoset CFRP-production was developed focusing the (diffusion-based) transfer reduction based on an adapted processing. Indicating the time in which the diffusion is possible, as the main criteria.
\end{abstract}

Keywords: Adhesive bonding, Contamination, Diffusion, Bonding pre-treatment

\section{Background}

The application of carbon-fiber reinforced plastics offers a high potential to decrease the weight of structural parts, without decreasing the performance of the product. Thus, the general and specific efficiency (e.g. eco-efficiency) is enlarged $[1,2]$. Unfortunately, there are significant challenges within the assembly respectively joining process of CFRPparts, especially if the matrix is a thermoset system. Conventional joining techniques cannot be applied due to its chemical composition (for example welding) or show significant disadvantages (mechanical joining e.g. riveting). The performance is decreased by the application of mechanical joints, since the elements cut the load bearing fibers or the fibers are cut by the priory performed drilling process. In addition a notch effect occurring around the rivet $[3,4]$. Compared with classical joints, adhesive bonding is therefore the more promising joining technique due to a more homogeneous load distribution and its capability to join thermoset and even different materials [5].

To provide a structural and durable bond, the adhesion between the CFRP-part and the adhesive has to be guaranteed [6], which is actually the most relevant challenge

(c) The Author(s) 2017. This article is distributed under the terms of the Creative Commons Attribution 4.0 International License (http://creativecommons.org/licenses/by/4.0/), which permits unrestricted use, distribution, and reproduction in any medium, provided you give appropriate credit to the original author(s) and the source, provide a link to the Creative Commons license, and indicate if changes were made. 
during the assembly process. The challenge is based on the fact, that independent from the specific manufacturing process, most of the CFRP-parts are produced in molds [7].

To guarantee the demolding of the cured parts, release agents are used. Within the aerospace industry (where thermoset matrix systems are widely used), external release agents are applied to coat the mold prior to the curing process [7]. After the crosslinking reaction of the resin-system and the demolding of the parts, release agent residues are found on the parts surface as several times shown (e.g. [6-9]). This indicates a release agent transfer during the production of the parts. These release agent residues lower the adhesion of the parts and therefore their initial bondability [10]. Within industrial applications, the challenge of the relatively bad initial bondability is solved by an additional process step-the bonding pre-treatment. Even if there are different approaches, like a laser [8] or plasma [9] application, which allow a sufficient and potentially automated bonding pre-treatment, it is still an additional process step which lowers the production efficiency.

To increase the production efficiency, an optimum of the "bonding quality" (as a parameter for the bondability) consisting out of "effort in terms of bonding pre-treatment", "contamination tolerant adhesive" and "surface cleanliness" respectively release agent transfer (see Fig. 1) has to be found. Those parameters are chosen because with a high level of every specific parameter a good bondability can be reached. For example, in the case that a very clean surface should be bonded, no bonding pre-treatment has to be performed (therefore its effort is small) and also the adhesive do not have to be contamination tolerant. Another example could be, that if an intense bonding pre-treatment was performed also surface which were not clean before can be bonded with a standard, non contamination tolerant adhesive.

To reduce the effort in terms of bonding pre-treatment the applied adhesives need to be more contamination tolerant or the release agent transfer has to be as low as possible to achieve a clean surface. Due to the fact, that there are no adhesives, which show the relevant tolerance for the typical release agents, the release agent transfer has to be lowered to increase the production efficiency. Unfortunately, the correlation between the

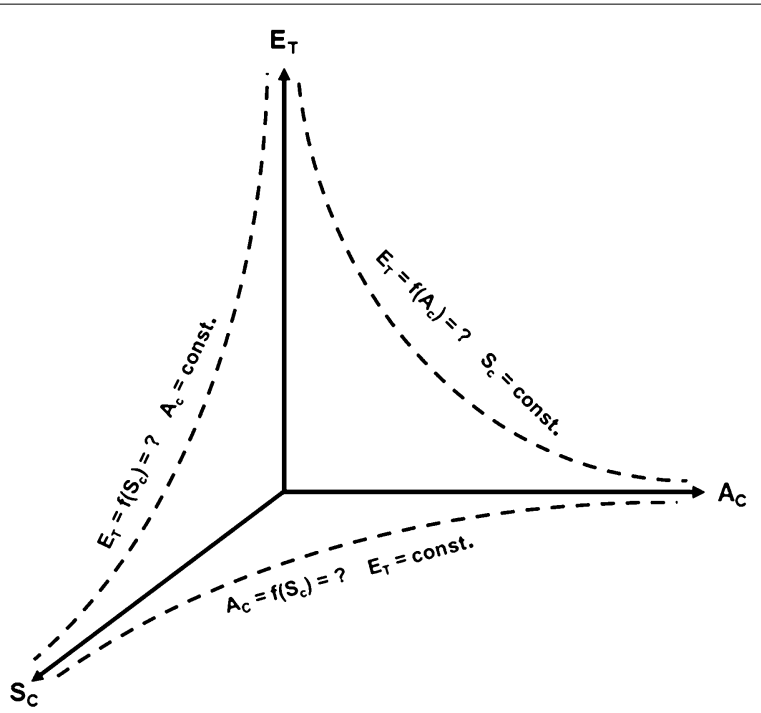

Fig. 1 "Bonding quality" as space spanned by effort in bonding pre-treatment $\left(E_{T}\right)$, surface cleanliness $\left(S_{C}\right)$ and contamination tolerant adhesive $\left(A_{C}\right)$ 
release agent transfer and the CFRP-part production process has not yet been investigated in literature.

However, there are some publications about a contamination tolerant bonding process of thermoset adhesives [11, 12]. During these investigations, the aim was to absorb as much contamination as possible in the adhesive to achieve an adequate bonding performance. This is mainly the inverse goal of the process presented in this study. As an approach, the models used for a contamination tolerant bonding process were inversely used and adapted with focus to the CFRP production and its process parameters to achieve the general goal, namely to subsequently reduce the release agent transfer by an adapted processing of the CFRP-system.

Therefore, the main objectives of this study were to identify the most relevant production parameter, which theoretically influence the release agent transfer and furthermore, to judge about their influence on this transfer. Based on the literature, this investigation shell be summarized to a qualitative model, which is the base for further investigations to finally increase the initial bondability of CFRP-parts.

\section{Fundamentals for the bonding performance of untreated CFRP}

Normally, the process parameters of a specific CFRP-production method (e.g. autoclave process) are chosen to achieve a high interlaminar quality [13] or to reach a fast part production [14]. Unfortunately, the correlation of specific process parameters on the release agent transfer has not been investigated so far and is therefore subsequently discussed.

During the presented approach, the release agents respectively its residues (e.g. silicone oils or silicone resins) on the mold can be seen as contaminations [15]. Within the autoclave process, the liquid matrix is interacting with these contamination layers, which represent a nonpolar surface.

Resulting, two aspects become relevant for the subsequent demoldability and consequently bonding performance of the demolded parts, namely the interaction of functional groups during the crosslinking reaction and the diffusion of contaminations inside the resin during its crosslinking process.

\section{Interaction of functional groups}

Besides the highly relevant diffusion of contaminations, one reason of the bad bondability of untreated demolded CFRP-parts is their nonpolar surface state. This nonpolar state can be explained by the orientation of the functional groups during the crosslinking reaction of the thermoset resins.

During this reaction, the functional groups of the uncured resin try to achieve the favorable energetic state [16]. In general, interactions with internal (within the resin) or external groups can be formed and are mainly influenced by the polar or nonpolar state of the functional groups. In epoxy resins, the molecules (especially ethers) can mainly be classified as negatively polar [16]. In the case of a positive polar environment (e.g. clean metal surface) and due to the energetic state, the functional groups of the resin interact with the metal surface in form of intermolecular bindings [16] resulting in a sufficient adhesion between metal and resin.

In the case of a nonpolar environment, (for example release agent coated molds), the interaction of these groups with the surface molecules would not lead to a favorable 
energetic state. Thus, the polar molecules are orientated towards the internal polymer network and the nonpolar molecules of this network are located towards the surface of the polymer [11]. This finally leads to the fact, that the surface of the cured resin respectively CFRP becomes nonpolar.

Finally, the same process becomes relevant during the subsequent bonding process. The nonpolar surface of the CFRP demolded out of release agent coated molds, only shows a few possible interaction points for the functional groups of the adhesive to create intermolecular bindings. Based on the state, that those bindings are the most relevant interactions for a sufficient adhesion, this results in a relatively low adhesion between CFRP and adhesive and therefore a poor bondability.

\section{Diffusion of contaminations}

Due to the fact, that release agents are often stoved on the molds prior to the first production cycle, a displacement (on a macro-or microscopic scale) of the contamination by the resin has a minor relevance. Therefore, the presence of release agent residues inside the top layer of CFRP-samples [17] has to be explained by diffusion processes, which is the most relevant process to justify the bad initial bondability.

This diffusion process is exemplarily shown in Fig. 2. At the beginning of the CFRPproduction process (Fig. 2a) the total amount of release agent $\left(\mathrm{N}_{0}\right)$ is distributed only on surface of the metallic mold (A), while no release agent is inside the resin. During the curing process the release agent molecules start to diffuse inside the resin. This leads to the fact, that at the end of the production process there is a gradient of release agent residues inside the cured resin (Fig. 2b). In the case, that $x$ describes their location perpendicular to the mold surface, it can be seen, that the contamination ratio $(C)$ of the

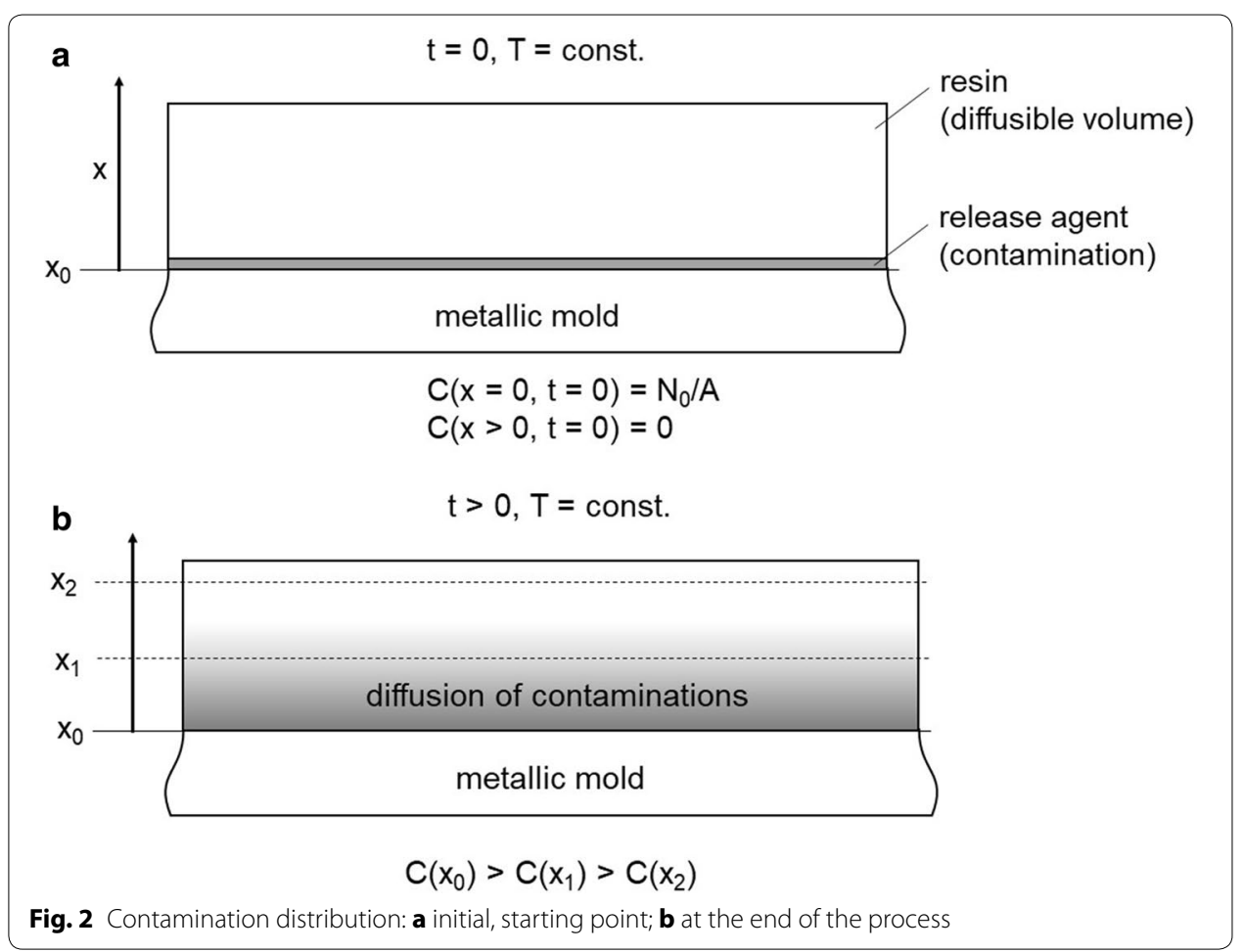


resin with release agent molecules decreases with increasing $x\left(C\left(x_{0}\right)>C\left(x_{1}\right)>C\left(x_{2}\right)\right.$ for $\left.\mathrm{x}_{0}<\mathrm{x}_{1}<\mathrm{x}_{2}\right)$.

So, the diffusion describes a diffusion flux (J) or an amount of particles resp. substances which flow through a unit area during a specific time unit. Based on the first Fick's law (Eq. 1) [18], this diffusion flux is proportional to the concentration gradient (shown by change of the concentration ratio over the location) with regard to a proportional factor $\mathrm{D}$-the diffusion coefficient.

$$
J=-D \frac{\partial C}{\partial x}
$$

With this equation it is possible to calculate a diffusion current, but due to the fact, that the concentration and with it the diffusion flux changes this model has to be enlarged to be able to calculate the global diffusion [18].

$$
\frac{\partial C}{\partial t}=-\frac{\partial J}{\partial x}
$$

Based on the assumptions that a mass preservation (Eq. 2) is given, meaning that the change of the concentration over the time is negatively proportional to the change of the diffusion flux over the location and the first Fick's law (Eq. 1), the diffusion equation can be defined to [18]:

$$
\frac{\partial C}{\partial t}=\frac{\partial}{\partial x}\left(D \frac{\partial C}{\partial x}\right)=D \cdot\left(\frac{\partial^{2} C}{\partial x^{2}}\right)
$$

The second Fick' law (Eq. 3) finally correlates the temporal and local change of the concentration. Based on the literature (cf. [19, 20]), the diffusion of contaminations (release agent molecules) inside the resin can be justified by the second Fick's law. In the specific case of this paper, the equation includes the contamination ratio at a specific location (x) perpendicular to the mold surface (cf. Fig. 2) and the diffusion coefficient (D) and describes a diffusion based on the local concentration difference between contamination (release agent) and resin (matrix) over the time $(\mathrm{t})$.

To reduce the contamination diffusion $(\delta)$ and therefore the release agent transfer, the diffusion coefficient has to be as small as possible during the production cycle until the gel point is reached $\left(t_{\text {gel }}\right)$ [21], because from this point on the established polymer network does not allow further diffusions afterwards. This finally leads to the following optimization problem (Eq. 4), namely the minimization of the integral.

$$
\Rightarrow \min \delta=\int_{0}^{t_{\text {gel }}} D d t
$$

Finally, by reducing the contamination diffusion, the surface of the CFRP will be more polar, because of the absence of nonpolar contaminations, which results in better bondability. 


\section{Theoretical considerations for CFRP-processing}

Besides the characteristics of the raw materials (e.g. release agent formulation, resin formulation or fillers), the release agent transfer is depending on the processing during the crosslinking reaction of the resin. This dependency is subsequently discussed to identify the different influences.

\section{Characteristics of the CFRP-production}

Due to the fact, that in the initial state the resin can be characterized as a fluid, the diffusion coefficient can be described based on the Stokes-Einstein equation. Thus, the diffusion coefficient for the contamination diffusion can be calculated (Eq. 5) by considering the temperature $(T)$, the Boltzmann constant $\left(k_{B}\right)$, the viscosity $(\eta)$ of the resin and the size of the diffusing particle, which can be described by a geometrical factor $(f)$ (cf. [11]).

$$
D_{S E}=D(T, f, \eta)=\frac{k_{B} \cdot T}{f \cdot \eta}
$$

Unfortunately, during the production of thermoset CFRP-parts the diffusion is hindered by the polymerization of the matrix resin. While for example higher temperatures decrease the viscosity of the resin, which favor the diffusion of contaminations, the polymerization is also accelerated by higher temperatures. Correlating, the time in which a diffusion is possible is decreased by higher temperatures, which results in the fact, that also the curing time is a function of the temperature.

Previous investigations [11] about the contamination diffusion relating to the contamination tolerant adhesive bonding proved a strong influence of the viscosity on the diffusion process and developed a model (Eq. 6) based on the time dependent temperature and viscosity.

$$
\delta=\int_{0}^{t_{\text {gel }}} \frac{k_{B} \cdot T(t)}{f \cdot \eta(t, T(t))} d t
$$

Due to the fact, that the time dependent gel point is often hard to measure resp. identify it is useful to use other approaches. One approach is given by Macosko and Miller [22]. They stated that due to probabilistic models of the average molecular weight $\left(\mathrm{m}_{\mathrm{w}}\right)$ the gel point for adhesives resp. resin with epoxy/amine basis is reached, when the molecular weight becomes infinite wich describes a characteristic crosslinking degree (X). Finally, the gel-point $\mathrm{X}_{\text {gel }}$ (Eq. 7) is depending on the ratio of the functional groups of epoxy- to amine hardener component ( $r$ ) and the functionality of the hardener component $\left(f_{a}\right)$

$$
X_{g e l}=\frac{1}{\left(r \cdot\left(f_{a}-1\right)^{1 / 2}\right.}
$$

Considering those thoughts, von Hayek-Boelingen [11] defined the model about the contamination diffusion as followed based on the crosslinking degree (Eq. 8):

$$
\int_{0}^{X_{g e l}} \frac{k_{B} \cdot T}{f \cdot \eta(X, T)} \cdot \frac{d t}{d X} d X
$$


While the time dependencies were considered in previous investigations about contamination tolerant bonding process, the influence of typical autoclave process parameters were neglected. Therefore, the model shown in Eq. (8) has to be extended by the process parameters autoclave pressure $(p)$ and the applied vacuum $(v)$.

This finally leads to the relevant question, namely how the process parameters time, temperature, autoclave vacuum $(v)$ and pressure $(p)$ (especially with their time dependencies) influence the polymer network with regard to a contamination diffusion until the gel point is reached.

\section{Influence of time}

As stated before, the diffusion of the contaminations inside the resin is due to a difference in terms of the concentration. Due to this fact, the diffusion is on one hand theoretically limited based on a leveled concentration, but on the other hand and for the given fabrication system the limitation can be neglected. Focusing the CFRP-part production the part thickness is significantly larger than the contamination layer thickness, which results into an almost infinite volume in which the contamination can diffuse.

Therefore, it is significant to reduce the time, in which a diffusion occurs. Based on the fact, that the diffusion is possible until the gel point is reached, the correlating gel time should be minimized (Eq. 9) to reduce the amount of diffused contaminations, resulting in a smaller contaminated resin volume.

$$
\Rightarrow \min t\left(X_{g e l}\right)
$$

\section{Influence of temperature and heating rate}

Concerning the release agent transfer respectively contamination diffusion, the temperature has two major aspects in its influence. On one hand the simple influence on the diffusion coefficient and on the other hand the influence on the viscosity have an impact.

Focusing the general approach for the description of the diffusion by a diffusion coefficient (see Eq. 2), it is obvious that an increased temperature leads to a higher diffusion coefficient, thus resulting into a thicker contamination layer on the CFRP-parts. Due to this fact, the temperature should be generally as small as possible until the gel point is reached to reduce the diffusion, without considering the higher crosslinking speed with higher temperatures.

Otherwise, the temperature has a significant influence on the viscosity, especially if prepreg-systems are used. Concerning the chosen approach (see Eq. 8), the viscosity should be as high as possible to reduce the contamination diffusion. Figure 3 shows the correlation between viscosity and temperature for a representative prepreg-system. In addition, three relevant phases are marked in the diagram, which are relevant for the release agent transfer.

In contrast, to conventional two-component adhesives or liquid resins, the viscosity of the matrix within prepreg semi-finished parts shows a significantly different behavior and therefore also the release agent transfer is influenced. At room temperature, the viscosity is relatively high and slowly decreasing while the temperature is increased. After a material specific temperature, the viscosity is relatively low and remains in this state for a relatively small temperature range. Further increases of the temperature lead to a sudden and significant increase of the viscosity. 


\section{Rheology}

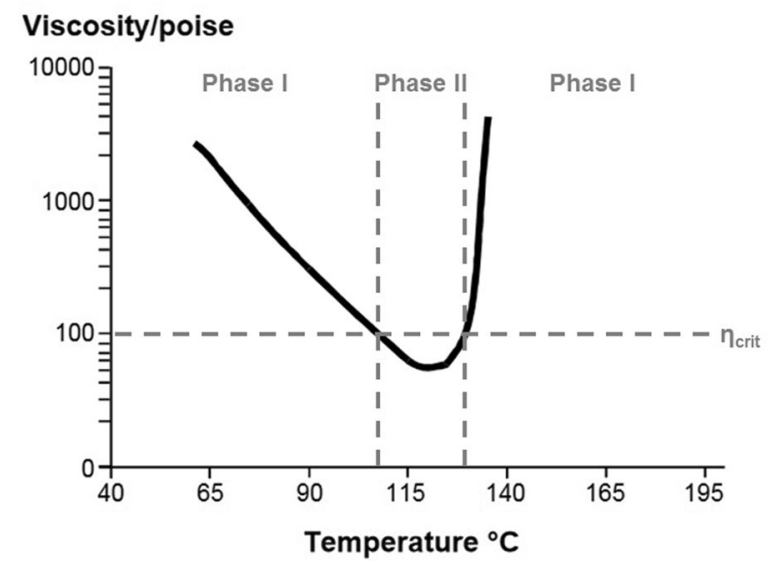

Fig. 3 Correlation of viscosity and temperature for a representative prepreg-system [23] with the schematically representation of critical viscosity and the relevant phases

Focusing the release agent transfer, three phases are relevant. With regards to the fact, that a diffusion is only technically relevant for a defined viscosity range below a critical viscosity $\left(\eta_{\text {crit }}\right)$ it has to be mentioned, that at the beginning of the CFRP-processing, the diffusion respectively the release agent transfer is not that crucial due to the high viscosity which is higher than the critical viscosity (phase I). Further increases in terms of the temperature lead to a further reduction of the viscosity until a plateau is reached (phase II). After the gel point is reached, the viscosity increases rapidly and nearly no more diffusion is possible (phase III). Even if the temperature range, in which the viscosity is below the critical viscosity, is relatively small, the amount of diffused contaminations can be reduced by the time horizon of this temperature range respectively the heating rate. Concluding, a high heating rate would reduce the amount of diffused contaminations.

To judge about the relevance of the previously mentioned aspect of an increased diffusion coefficient by an increased temperature and the reduced diffusion due to the viscosity-profile, the crosslinking process of the epoxy resin has to be considered.

Due to the ongoing crosslinking reaction, an increase of the temperature leads to a significant reduction of the gel time (see Fig. 4) in which the diffusion is possible. In contrast, an increase of the temperature do not favor the diffusion this strongly [11].

Based on these conclusions and desiring a minimum amount of release agent transfer, the authors of the present study suggest, to increase the heating rate and the temperature due to the reduced gel time, with regards to material specific temperatures (e.g. decomposition temperature).

$$
\Rightarrow \max \int_{0}^{t_{g e l}} T(t) d t
$$

\section{Influence of autoclave vacuum}

During autoclave processes, a vacuum is applied to the CFRP-parts (typically more than -0.8 bars compared to the atmosphere [25]). This is mainly done, to increase the 


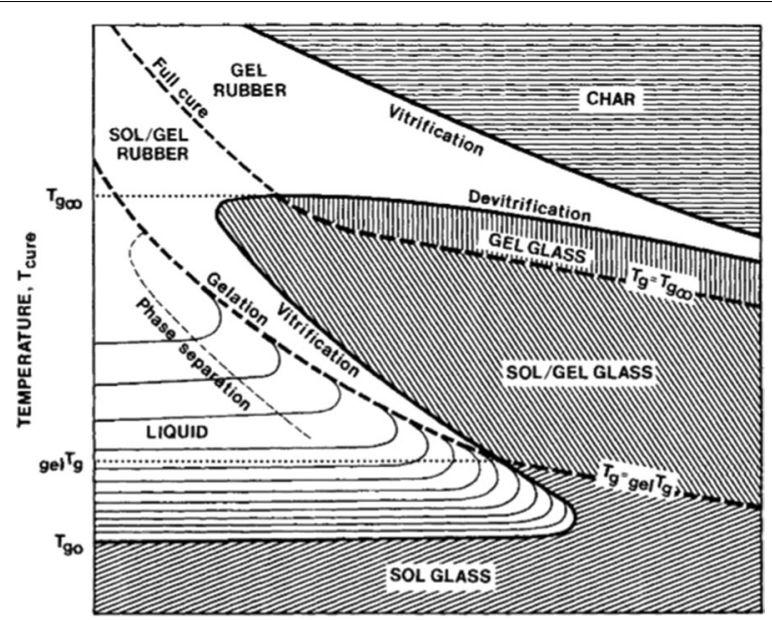

LOG TIME

Fig. 4 Generalized time-temperature-transformation (TTT) diagram [24]

interlaminar quality of the parts $[13,26]$ (e.g. to reduce the amount of voids inside of the part [27]). Nevertheless, in theory the applied vacuum also has an impact on the release agent transfer.

Therefore, it has to be considered, that within a vacuum the atmosphere varies significantly compared to normal conditions. Especially two aspects become relevant-at first the absence of oxygen and second the absence of moisture in comparison to the ambient atmosphere.

In general, at ambient atmosphere the presence of oxygen lead to the possibility for interactions between the molecules of the resin or the hardener, which could be very specific reactions for a specific material selection.

Focusing the impact of moisture on the polymer network within epoxy systems, a closer look on those systems has to be done. Within typical epoxy systems the epoxy resin react within a polyaddition with an amine hardener [28]. Unfortunately, the amine hardeners are hygroscopic and have the tendency to react within side reactions with water [29], for example shown by Sanjana et al. [30] for the reactivity of an epoxy based prepreg.

Resulting, both effects (the presence of oxygen and the presence of moisture) lead to side reaction within the crosslinking process of the epoxy. Those side reactions bind reaction partners for the original reactions. This lead to an unfavorable share of hardener to resin components and therefore in a slower curing [31] due to a reduced number of reaction starting points, which finally increases the gel time.

In addition, the vapor pressure of the release agent could also be relevant, if silicone oils are parts of the applied release agent. At higher temperatures, those silicone oils have a relatively low vapor pressure [32], which could be critical for the CFRP-processing and would may be indicate a negative effect of an applied vacuum on the release agent transfer. Nevertheless, even if the vapor pressure is reached during the production cycle, the volatile components of the release agent are mainly sucked outside of the laminate by the applied vacuum system within the experimental set-up (e.g. by the application of 
vacuum bags $[27,33])$, proven by the reduction of voids after the application of an higher vacuum [13].

Due to these effects, a diffusion of contamination molecules or particles is favored, if the reaction is performed under normal atmosphere. Therefore, the applied vacuum should be as high as possible (Eq. 11) to reduce the amount of side reactions until the gel point is reached.

$$
\Rightarrow \max \int_{0}^{t_{\text {gel }}} v(t) d t
$$

\section{Influence of autoclave pressure}

Besides processing time, temperature and autoclave vacuum, the autoclave pressure is the last relevant process parameter within CFRP-part production in aerospace production lines. Generally, the autoclave pressure guarantees in combination with the applied vacuum the interlaminar quality of the CFRP-parts and is varied up to 8 bars [33, 34].

Focusing the release agent transfer three aspects become relevant due to the application of an additional pressure inside the autoclave. Those aspects are at first the increase of the viscosity [35], the reduction of the free-volume within the resin and a better contact between the resin and the contamination layer due to an increased pressure [36].

One model to describe the viscosity of a polymer during its crosslinking reaction is the free volume theory by Williams, Landel and Ferry [37]. Within this theory, the viscosity is determined by the volume of the resin, which is not occupied by the atoms of the polymer chains [38]. During the crosslinking reaction, the polymer chain is more and more enlarged, and therefore the free volume is reduced, which increases the viscosity [39] due to a reduced mobility of the molecules [40]. Unfortunately, there are no investigations which directly deal with the influence of an additional pressure on the viscosity of an epoxy resin, but in theory, there is an increase of the viscosity, due to an applied pressure [37]. This effect is also justified by the reduction of the free volume due to the additional pressure and the following reduced mobility of the molecules.

In theory, the reduction of the free volume within the liquid resin has another impact on the diffusion. The free volume within the resin also represents space, through which the contaminations can diffuse easier [41]. Therefore, if the free volume is reduced, also the infusibility of the resin by the contamination molecules is hindered.

The third aspect of the impact of an increased autoclave pressure is the closer interaction respectively an enlarged contact surface between the contaminations and the resin. An additionally applied pressure leads to the facts, that on the one hand, the resin molecules are forced towards to the contamination layer and on the other hand, the surface wetted by the resin is increased, because the resin is pressed also in small pores [16].

Focusing, the shorter distance between the resin and the contaminations, the diffusion paths to reach a specific penetration depth are also smaller. Concerning the significance for the release agent transfer, this is relevant, since for a given gel time this leads to a thicker contamination layer which subsequently leads to a higher effort in terms of bonding pre-treatment to achieve the sufficient cleanliness of the surface.

Comparing the impact of the three aspects, the authors assume, that a noticeable increase of the viscosity and the decrease of the free volume due to an increased 
pressure require a significant higher pressure (more than one magnitude) than realized within an autoclave process, as for example shown by Radusch et al. [42] for thermoplastics. Therefore, the third aspect is characterized as the relevant one, which lead to the conclusion, that the applied pressure should be as small as possible (Eq. 12) to achieve the cleanest surface state with the least amount of release agent residues, which limits the adhesion.

$$
\Rightarrow \min \int_{0}^{t_{g e l}} p(t) d t
$$

\section{Discussion of the developed model}

As mentioned before, the cleanliness of the CFRP surface after demolding should be as high as possible to reduce the effort in terms of bonding pre-treatment respectively to allow an initial durable bonding of those parts. Therefore, the release agent transfer $\left(\delta_{\text {rat }}\right)$ during the part production should be as small as possible.

With regard to the positive effects of a reduced gel time ("Influence of time" section), an increased temperature ("Influence of temperature and heating rate" section), an increased vacuum ("Influence of autoclave vacuum" section) and a decreased autoclave pressure ("Influence of autoclave pressure" section), the process-parameter based contamination diffusion release agent transfer $\left(\delta_{\mathrm{p}}\right)($ Eq. 13) can be defined to:

$$
\delta_{p}=t \cdot D_{p}(T(t), v(t), p(t))
$$

The diffusion coefficient for the process-parameter based release agent transfer $\left(D_{p}\right)$ can be stated as shown in Eq. (14):

$$
D_{p}(T(t), v(t), p(t))=\frac{p(t)}{T(t) \cdot v(t)}
$$

Within this equation, all parameters which increase the release agent transfer with an enlarged value (time and pressure) are inserted in the numerator, while the parameters which decrease the release agent transfer with higher value are inserted in the denominator.

With this definition and Eq. (5) for the general calculation of the diffusion coefficient (Stokes-Einstein equation), the diffusion coefficient for the release agent transfer can defined by the following equations (Eq. 15 resp. Eq. 16)

$$
\begin{aligned}
& D_{\text {rat }}=D_{S E} \cdot D_{p} \\
& D_{\text {rat }}=\frac{k_{b} \cdot T(t)}{f \cdot \eta} \cdot \frac{p(t)}{T(t) \cdot v(t)}
\end{aligned}
$$

Focusing Eq. (16) it can be seen, that the temperature is influencing as well the numerator as the denominator. Due to the fact, that the influence within the second part of the equation (representing) $D_{p}$ the temperature influence is representing the temperatureinfluenced viscosity (see "Influence of temperature and heating rate" section), this factor can be condensed with the factor of the viscosity, resulting in the final definition of the diffusion coefficient for the release agent transfer (Eq. 17). 


$$
D_{\text {rat }}=\frac{k_{b} \cdot T(t)}{f \cdot \eta(t, T(t), p(t))} \cdot \frac{p(t)}{v(t)}
$$

Considering this thoughts and Eq. (2) this finally leads to the objective function (Eq. 18).

$$
\min \delta_{\text {rat }}=\int_{0}^{t_{\text {gel }}} \frac{k_{B} \cdot T(t)}{f \cdot \eta(t, T(t), p(t))} \cdot \frac{p(t)}{v(t)} d t
$$

Even if all mentioned process parameters have an influence on the diffusion coefficient respectively the release agent transfer, the crucial point is to reduce the time in which the contamination diffusion is possible.

This can be realized by different approaches, even if the diffusion does not have a high relevance for high viscosities (correlating with room temperature for the focused prepreg systems), it should be considered that there is always a small diffusion of contamination particles, once the prepreg is in contact with the mold surface respectively the contamination layer. Resulting and if possible, the laminate lay-up should be performed outside the coated mold, to initially reduce the contamination diffusion. Besides, and once the laminate is within the mold, the most effective way to decrease the gel time is to increase the temperature and the heating rate with regard to the material damaging temperatures. As stated above, the slightly increased mobility of the contamination due to an increased heating rate and temperature is more than leveled by the reduced contact time and thus the reduced gel time.

Furthermore, a highly reactive prepreg system should be used to reach a fast crosslinking of the resin. Besides the material formulation, the reactivity can also be influenced by the processing prior and within the autoclave. Prior to the autoclave, the prepreg should be stored at low temperatures, to reduce the amount of side reaction due to some prepreg aging effects. Furthermore the laminate lay-up should be performed at conditions, which do not favor side reactions (such as relatively low temperature and relatively low moisture content) and the usage of in between vacuum applications of the layup will decrease the content of trapped interlaminar side reactants (e.g. trapped air). Within the autoclave, the amount of side reactions can be reduced by the application of a vacuum, which should be as high as possible to reduce moisture and oxygen content within the vacuum bag. In addition, the vacuum finally increases the interlaminar part quality.

\section{Conclusions}

The process parameters during CFRP-part production are mainly chosen to increase the interlaminar quality or due to a fast production rate. Besides those aspects, the parameters can also potentially influence the part quality in terms of the contamination of its surface due to mold release agent residues. The presented correlations show, that there is a significant potential to influence the release agent transfer during the production of fiber reinforced parts by an adapted application of specific process parameters. Based on the theoretical interactions of the process parameters time, temperature, vacuum and autoclave pressure with the contamination diffusion on the one hand and the polymer crosslinking on the other, a theoretical, qualitative model about the influence could be established. 
Even if initial experimental results seem to underline the validity of the presented approach and the established model, further work has to be done to finally prove the full validity for different production processes and different materials to develop optimized production cycles to reduce the release agent transfer. Furthermore, the need to establish correction factors to establish the quantitative model has to be proven. In addition, the impact of these optimized production cycles on the CFRP performance has to be investigated.

\begin{abstract}
Abbreviations
A: mold surface; $A_{c}$ : contamination tolerant adhesive; $C$ : concentration ratio; $C(x)$ : contamination concentration at a specific location $x$; CFRP: carbon fiber reinforced plastics; $D$ : diffusion coefficient; $D_{p}$ : $D$ for the process-parameter based release agent transfer; $D_{\text {rat }}$ D for the release agent transfer; $D_{S E}$ : D obtained out of the Stokes-Einstein equation; $\delta$ : contamination diffusion; $\delta_{p}: \delta$ only due to the process parameters; $\delta_{\text {rat }}$ : release agent transfer; $E_{T}$ : effort in terms of bonding pre-treatment; $f$ : geometrical factor of diffusing particles; $f_{a}$ : functionality of the amine hardener; $J$ : diffusion flux; $k_{B}$ : Boltzmann constant; $m_{w}$ : average molecular weight; $N_{0}$ : total amount of release agent; $\eta$ : viscosity; $p$ : autoclave pressure; $r$ : ratio of epoxy/amine content; $S_{C}$ : surface cleanliness; $T$ : temperature; $t$ : time; $t_{\text {gel }}$. gel point (time based); TTT: time-temperature-transformation; $v$ : autoclave vacuum; $X$ : crosslinking degree; $X_{g e l}$ : gel point (crosslinking based); $x$ : Iocation.
\end{abstract}

\title{
Authors' contributions
}

DB developed the presented model based on a literature review. This model was cross-checked by SH and KD. All authors read and approved the final manuscript.

\section{Acknowledgements}

The presented results were found within the project "Vorteil" (Funding Code KF2012222TA4) sponsored by the German Federal Ministry for Economic Affairs and Energy (BMWi) on the basis of a decision of the German parliament. The financial support is gratefully acknowledged. In addition the authors would like to thank the project partner Invent GmbH, Braunschweig, Germany for the supportive and excellent collaboration.

\section{Competing interests}

The authors declare that they have no competing interests.

\section{Consent for publication}

The authors consent to the publication process.

Ethics approval and consent to participate

The authors consent to the ethics.

\section{Funding}

The research (Funding Code KF2012222TA4) was funded by the German Federal Ministry for Economic Affairs and Energy (BMWi) on the basis of a decision of the German parliament.

\section{Publisher's Note}

Springer Nature remains neutral with regard to jurisdictional claims in published maps and institutional affiliations.

Received: 17 August 2017 Accepted: 20 September 2017

Published online: 25 September 2017

\section{References}

1. Lässig R, Eisenhut M, Mathias A, et al. Serienproduktion von hochfesten Faserverbundbauteilen-Perspektiven für den deutschen Maschinen und Anlagenbau. Frankfurt am Main: VDMA Verlag; 2012.

2. Fuchs ERH, Field FR, Roth R, et al. Strategic materials selection in the automobile body: economic opportunities for polymer composite design. Compos Sci Technol. 2008;68(9):1989-2002. doi:10.1016/j.compscitech.2008.01.015

3. Darwish F, Tashtoush G, Gharaibeh M. Stress concentration analysis for countersunk rivet holes in orthotropic plates. Eur J Mech A-Solid. 2013;37:69-78. doi:10.1016/j.euromechsol.2012.04.006.

4. Heredia FE, Spearing SM, Mackin TJ, et al. Notch effects in carbon matrix composites. J Am Ceram Soc. 1994;77(11):2817-27. doi:10.1111/j.1151-2916.1994.tb04510.x.

5. Budhe S, Banea MD, de Barros S, et al. An updated review of adhesively bonded joints in composite materials. Int J Adhes Adhes. 2017;72:30-42. doi:10.1016/j.jijadhadh.2016.10.010

6. Chin J, Wightman J. Surface characterization and adhesive bonding of toughened bismaleimide composites. Compos Part A Appl Sci Manuf. 1996;27(6):419-28. doi:10.1016/1359-835X(95)00064-9 .

7. Campbell FC. Manufacturing technology for aerospace structural materials. Aerospace engineering materials science. 1st ed. Amsterdam: Elsevier; 2006. 
8. Fischer $F$, Kreling $S$, Jäschke $P$, et al. Laser surface pre-treatment of CFRP for adhesive bonding in consideration of the absorption behaviour. J Adhes. 2012;88(4-6):350-63. doi:10.1080/00218464.2012.660042 .

9. Kusano Y. Atmospheric pressure plasma processing for polymer adhesion: a review. J Adhes. 2014;90(9):755-77. doi: 10.1080/00218464.2013.804407.

10. Parker BM, Waghorne RM. Surface pretreatment of carbon fibre-reinforced composites for adhesive bonding. Composites. 1982;13(3):280-8. doi:10.1016/0010-4361(82)90011-8.

11. Hayek-Boelingen Mv. Wege zum kontaminationstoleranten Kleben. PhD Thesis, Neubiberg; 2004.

12. Holtmannspötter J, Czarnecki J, Gudladt H-J. The use of power ultrasound energy to support interface formation for structural adhesive bonding. Int J Adhes Adhes. 2010;30(3):130-8. doi:10.1016/j.ijadhadh.2009.10.002.

13. Koushyar H, Alavi-Soltani S, Minaie B, et al. Effects of variation in autoclave pressure, temperature, and vacuumapplication time on porosity and mechanical properties of a carbon fiber/epoxy composite. J Compos Mater. 2012;46(16):1985-2004. doi:10.1177/0021998311429618.

14. Khan LA, labal Z, Hussain ST, et al. Determination of optimum cure parameters of 977-2A carbon/epoxy composites for quickstep processing. J Appl Polym Sci. 2013;129(5):2638-52. doi:10.1002/app.38990 .

15. Baldan A. Adhesively-bonded joints and repairs in metallic alloys, polymers and composite materials: adhesives, adhesion theories and surface pretreatment. J Mater Sci. 2004;39(1):1-49. doi:10.1023/B:JMSC.0000007726.58758. e4.

16. Habenicht G. Kleben: grundlagen, technologien, anwendungen, 6, aktualisierte Aufl. VDI-Buch. Berlin: Springer; 2009.

17. Kreling S, Fischer F, Delmdahl R, et al. Analytical characterization of CFRP laser treated by excimer laser radiation. Phys Procedia. 2013;41:282-90. doi:10.1016/j.phpro.2013.03.080.

18. Vieth WR. Diffusion in and through polymers: principles and applications; with 17 tables. München: Hanser; 1991.

19. Fink BK, McCullough RL. Interphase research issues. Compos Part A Appl Sci Manuf. 1999;30(1):1-2. doi:10.1016/ S1359-835X(98)00118-3

20. Shanahan MER. Diffusion effects in the wetting of a contaminated surface. J Colloid Interface Sci. 2000;229(1):16873. doi:10.1006/jcis.2000.6996.

21. Gillham JK. Award address formation and properties of network polymeric materials. Polym Eng Sci. 1979;19(10):676-82. doi:10.1002/pen.760191005.

22. Macosko CW, Miller DR. A new derivation of average molecular weights of nonlinear polymers. Macromolecules. 1976;9(2):199-206. doi:10.1021/ma60050a003 .

23. Hexcel. HexPly ${ }^{\circledR} 913-125^{\circ} \mathrm{C}$ curing epoxy matrix (Product Data Sheet); 2016.

24. Gillham JK. Formation and properties of thermosetting and highTg polymeric materials. Polym Eng Sci. 1986;26(20):1429-33. doi:10.1002/pen.760262012.

25. Boey FYC, Lee TH, Sullivan PL. High-pressure autoclave curing for a thermoset composite: effect on the glass transition temperature. J Mater Sci. 1994;29(22):5985-9. doi:10.1007/BF00366883.

26. Sudarisman Davies IJ. Influence of compressive pressure, vacuum pressure, and holding temperature applied during autoclave curing on the microstructure of unidirectional CFRP composites. Adv Mater Res. 2008;41-42:323-8. doi:10.4028/www.scientific.net/AMR.41-42.323

27. Boey F, Lye SW. Effects of vacuum and pressure in an autoclave curing process for a thermosetting fibre-reinforced composite. J Mater Process Technol. 1990;23(2):121-31. doi:10.1016/0924-0136(90)90152-K.

28. Ehlers J-E, Rondan NG, Huynh LK, et al. Theoretical study on mechanisms of the epoxy?: amine curing reaction. Macromolecules. 2007:40(12):4370-7. doi:10.1021/ma070423m

29. Reimers J, Ziegmann G. Moisture absorption of an amine curing agent and the influence on the properties of cured epoxy resins. J Plast Technol. 2012;8(2):75-90

30. Sanjana ZN, Schaefer WH, Ray JR. Effect of aging and moisture on the reactivity of a graphite epoxy prepreg. Polym Eng Sci. 1981;21(8):474-82. doi:10.1002/pen.760210807 .

31. Collong W, Göbel A, Kleuser B, et al. 2 K waterborne clearcoat?: a competition between crosslinking and side reactions. Prog Org Coat. 2002;45(2-3):205-9. doi:10.1016/50300-9440(02)00052-8.

32. Wilcock DF. Vapor pressure-viscosity relations in methylpolysiloxanes. J Am Chem Soc. 1946;68(4):691-6. doi:10.1021/ja01208a050.

33. Boey F, Lye SW. Void reduction in autoclave processing of thermoset composites. Composites. 1992;23(4):261-5. doi:10.1016/0010-4361(92)90186-X.

34. Blass $D$, Kreling $S$, Dilger $K$. The impact of prepreg aging on its processability and the postcure mechanical properties of epoxy-based carbon-fiber reinforced plastics. Proc IME J Mater Des Appl. 2017;231(1-2):62-72. doi:10.1177/1464420716665413

35. Ferry JD, Stratton RA. The free volume interpretation of the dependence of viscosities and viscoelastic relaxation times on concentration, pressure, and tensile strain. Kolloid-Z. 1960;171(2):107-11. doi:10.1007/BF01520041.

36. Hong SG, Boerio FJ. Assimilation of oil from metal surfaces by epoxy adhesives: XPS and ATR analyses. J Appl Polym Sci. 1995;55(3):437-49. doi:10.1002/app.1995.070550309

37. Ferry JD. Viscoelastic properties of polymers. 3rd ed. New York: Wiley; 1980.

38. Mark JE. Physical properties of polymer handbook. 2nd ed. New York: Springer; 2007.

39. Sanford WM, McCullough RL. A free-volume-based approach to modeling thermoset cure behavior. J Polym Sci B Polym Phys. 1990;28(7):973-1000. doi:10.1002/polb.1990.090280701 .

40. Lapique F, Redford K. Curing effects on viscosity and mechanical properties of a commercial epoxy resin adhesive. Int J Adhes Adhes. 2002;22(4):337-46. doi:10.1016/50143-7496(02)00013-1 .

41. Ratna D. Handbook of thermoset resins, 1. publ. Handbook Ser. iSmithers, Shawbury, Shrewsbury: 2009

42. Radusch H-J, Kies T, Altenbach $\mathrm{H}$, et al. Influences concerning the properties of small and thinwalled injection molded parts. J Plast Technol. 2006;2(6):1-21. 